\title{
Intensive upper limb neurorehabilitation in chronic stroke: outcomes from the Queen Square programme
}

\author{
Nick S Ward, ${ }^{\oplus 1,2,3}$ Fran Brander, ${ }^{2,3}$ Kate Kelly ${ }^{2,3}$
}

\begin{abstract}
- Additional material is published online only. To view please visit the journal online (http://dx.doi.org/10.1136/ jnnp-2018-319954).
\end{abstract}

${ }^{1}$ Department of Clinical and Motor Neuroscience, UCL Institute of Neurology, London, UK

${ }^{2}$ The National Hospital for Neurology and Neurosurgery, London, UK

${ }^{3}$ UCLP Centre for

Neurorehabilitation, London, UK

\section{Correspondence to}

Professor Nick S Ward Department of Clinical and Motor Neuroscience, UCL Institute of Neurology, London WC1N 3BG, UK; n.ward@ucl. ac.uk

Received 5 November 2018 Revised 21 January 2019 Accepted 28 January 2019 Published Online First 15 February 2019

\begin{abstract}
Objective Persistent difficulty in using the upper limb remains a major contributor to physical disability post-stroke. There is a nihilistic view about what clinically relevant changes are possible after the early post-stroke phase. The Queen Square Upper Limb Neurorehabilitation programme delivers high-quality, high-dose, high-intensity upper limb neurorehabilitation during a 3-week (90 hours) programme. Here, we report clinical changes made by the chronic stroke patients treated on the programme, factors that might predict responsiveness to therapy and the relationship between changes in impairment and activity.

Methods Upper limb impairment and activity were assessed on admission, discharge, 6 weeks and 6 months after treatment, with modified upper limb Fugl-Meyer (FM-UL, max-54), Action Research Arm Test (ARAT, max-57) and Chedoke Arm and Hand Activity Inventory (CAHAl, max-91). Patient-reported outcome measures were recorded with the Arm Activity Measure (ArmA) parts $A(0-32)$ and $B(0-52)$, where lower scores are better.

Results 224 patients (median time post-stroke 18 months) completed the 6-month programme. Median scores on admission were as follows: FM-UL = 26 (IQR 16-37), ARAT =18 (IQR 7-33), CAHAI=40 (28-55), ArmA-A=8 (IQR 4.5-12) and ArmA-B=38 (IQR 24-46). The median scores 6 months after the programme were as follows: FM-UL=37 (IQR 24-48), ARAT=27 (IQR 12-45), CAHAI=52 (IQR 35-77), ArmA-A=3 (IQR 1-6.5) and $A r m A-B=19$ (IQR 8.5-32). We found no predictors of treatment response beyond admission scores.

Conclusion With intensive upper limb rehabilitation, chronic stroke patients can change by clinically important differences in measures of impairment and activity. Crucially, clinical gains continued during the 6-month follow-up period.
\end{abstract}

\section{INTRODUCTION}

Stroke remains common ${ }^{1}$ and persistent difficulty in using the upper limb is a major contributor to ongoing physical disability. ${ }^{2}$ The general consensus remains that most spontaneous recovery of the upper limb occurs over the first 3 months after stroke and current levels of rehabilitation result in little improvement after that, particularly at the level of impairment. ${ }^{3}$ Improving outcomes through higher dose (time in rehabilitation or number of repetitions) and intensity (dose per session) of rehabilitation is an attractive option. ${ }^{4}$ However, clinical trials of higher dose upper limb rehabilitation have generally not produced the magnitude of improvement that will change clinical practice, ${ }^{5}$ whether delivered in the early ${ }^{6}$ or chronic stages post-stroke. ${ }^{7-9}$ A common factor in these trials is that the dose (in hours) of additional therapy remained relatively low (18-36 hours). Despite scepticism that stroke patients could tolerate much higher doses, ${ }^{8}$ one study managed to deliver 300 hours of upper limb therapy to chronic stroke patients over 12 weeks and reported changes in measures of both impairment and activity that were far greater than those in lower dose studies. ${ }^{10}$ Three hundred hours represents an order of magnitude higher than any dose of rehabilitation offered in previous upper limb rehabilitation trials and deserves further consideration. However, this idea is challenging because of the logistics of setting up such a trial in healthcare settings where the ethic of high-dose, high-intensity rehabilitation is not supported. In this context, it is important to report the findings of clinical services that are able to deliver higher doses than conventionally seen. The Queen Square Upper Limb (QSUL) Neurorehabilitation programme is a single-centre clinical service that provides 90 hours of timetabled treatment focusing on the post-stroke upper limb in chronic (>6 months post-stroke) stroke patients. Here, we report (i) outcomes for patients admitted to this programme at the National Hospital for Neurology and Neurosurgery, University College London Hospitals NHS Trust (UCLH), including 6-month follow-up data to look at whether any clinical benefits were maintained, (ii) the characteristics of the patients admitted and any predictors of response and (iii) the relationship between changes in impairment and activity.

\section{METHODS}

\section{Participants}

Patients were referred by primary care physicians for advice about ongoing management of the paretic upper limb following stroke. There were no criteria around time since stroke. Clinical criteria warranting admission to the programme were broad but were focused on whether we felt able to help patients achieve their goals for the affected upper limb. Barriers to admission included factors precluding the ability to work in upper limb tasks: (i) absent movements, throughout the limb; (ii) a painful shoulder limiting an active forward reach (mostly due to adhesive capsulitis); (iii) severe spasticity or non-neural loss of range and (iv) unstable medical conditions. These patients were not offered admission to the treatment programme but were provided with appropriate advice or referred for 
other treatment approaches. Re-referral to the programme was then considered (eg, after successful management of adhesive capsulitis of the shoulder). Features that were more likely to result in admission to the programme included at least some ability to flex the shoulder and at least visible flickers of movement in finger and/or wrist extensors, although this was not always the case as reflected in baseline upper limb scores. Patients were admitted to the programme as day attenders, either from home or from the UCLH-dedicated patient accommodation and as such were either self-caring or self-caring with the support of one other person.

\section{Interventions}

Initial assessment consisted of analysis of both movement and performance in activities of daily living. Subsequent treatment was aimed at reducing impairment and promoting re-education of motor control within activities of daily living. Individualised meaningful tasks were practiced repeatedly in order to facilitate task mastery with a focus on quality of movement. This was achieved through (i) adaptation of the task, for example, decomposing tasks into individual components to be practiced; (ii) adaptation of the environment, for example, fabrication of functional splints and adaptation of tools such as cutlery or screwdrivers, to enable integration of the affected hand in meaningful activities; (iii) assistance, for example, de-weighting the arm to allow strengthening and training of movement quality and control through increased range and (iv) independent task practice. Coaching was considered a key component of the programme and used throughout to embed new skills and knowledge into individual daily routines. Consequently, individuals increase participation and confidence in their desired goals, enhancing self-efficacy and motivation to sustain behavioural change beyond the end of the active treatment period.

This overall approach was achieved through two daily sessions each of physiotherapy and occupational therapy, supplemented with tailored, individualised interventions, including repetitive practice with a rehabilitation assistant or robotic device, sensory retraining, use of dynamic and functional orthoses, neuromuscular electrical stimulation and group work. Furthermore, patients were encouraged to work on cardiovascular fitness during the programme. A 6-hour timetable was implemented 5 days a week for 3 weeks (total therapy time, 90 hours). Motor tasks could be described as passive or active, assisted or unassisted, and functional or nonfunctional. Over the 3 weeks, the aim was to increase the time spent on active, unassisted functional tasks, depending on a patient's level of impairment and progress. The programme was staffed with a 1:1 staff to patient ratio (two physiotherapists, two occupational therapists and two rehabilitation assistants for six patients).

\section{Quantitative assessment}

All patients had the following baseline scores measured on admission (table 1): modified Rankin scale (mRS), Barthel Index (BI), Neurological Fatigue Index (NFI), Hospital Anxiety and Depression Scale (HADS) and sensation (as indexed by light touch on palm as described in Fugl-Meyer, FM, assessment of the upper extremity).

The affected upper limb was assessed on admission (T1), discharge (T2), 6 weeks (T3) and 6 months (T4) postdischarge, using the following measures: FM (upper limb) (FM-UL), Action Research Arm Test (ARAT), Chedoke Arm and Hand Activity Inventory (CAHAI-13) and Arm Activity Measure (ArmA).
The FM-UL is a stroke-specific, performance-based impairment index (where impairment refers to loss of body structure and function) with good validity and intra-rater and inter-rater reliability. ${ }^{11} 12$ Here, we have used a modified version of the FM-UL, excluding measures of coordination and reflexes based on hierarchical properties of the scale, ${ }^{13}$ and as these do not relate to the upper limb synergies of interest. ${ }^{14}$ The minimum clinically important difference (MCID) has been reported as 5.25 points. ${ }^{15}$

The ARAT assesses a patient's ability to handle objects differing in size, weight and shape and therefore can be considered to be an arm-specific measure of activity limitation. ${ }^{16}$ The MCID has been suggested as 5.7 points. $^{17}$

The CAHAI-13 is a validated, upper limb measure that uses a 13-point quantitative scale in order to assess recovery of the arm and hand in performing activities of daily living after a stroke. ${ }^{18}$ It is a performance test using day-to-day items. It is not designed to measure the patient's ability to complete the task using only their unaffected hand, but rather to encourage bilateral upper limb use. No MCID has been reported for CAHAI-13 (although the minimum detectable change is reported as $6.2^{18}$ ).

The ArmA is a patient-reported outcome score ${ }^{19}$ with two components. The ArmA-A asks patients about their ability to care for their affected arm either themselves with their unaffected arm or by a carer or a combination of both of these. ArmA-B asks patients about how easy or hard it is to use the affected arm in activities of daily living. For ArmA-A and ArmA-B, note that lower scores are better. No MCID has been reported for ArmA-A or ArmA-B.

\section{Analysis plan}

Our primary aim was to measure changes in upper limb deficit over time which were examined using the Friedman test for all outcome scores. Post-hoc analysis to test differences between individual time points was carried out with the Wilcoxon signedrank test. As a secondary aim, we were then interested in whether baseline characteristics correlated with final (T4) outcomes. We initially examined for correlations between individual baseline characteristics and outcomes using Spearman's rank correlation. We also tested whether median T4 outcomes were different depending on gender or side of lesion using Wilcoxon rank sum test. Time since stroke was not considered as a covariate as once in the chronic phase, there is no indication that time since stroke has a linear effect on outcome. Lastly, we performed multiple linear regression to look for predictors of either absolute scores at T4 or the change in scores T1 -T4. Lastly, we also examined the relationship between the changes in outcome scores from admission to 6-month follow-up using Spearman's rank correlation.

\section{RESULTS}

\section{Baseline characteristics}

Between January 2015 and December 2017, 268 patients were admitted to the QSUL programme (representing 46\% of patients assessed in out-patient clinic). $40.4 \%$ of patients were admitted from home while $59.6 \%$ stayed in the UCLH-dedicated patient accommodation. Note, only 16 patients were admitted to the programme prior to 6 months post-stroke, and only one patient prior to 3 months, reflecting overall referral patterns to the programme.

Of those, 30 were non-stroke (12 traumatic brain injury, 8 brain tumour, 3 peripheral neurological conditions, 3 spinal cord injury and 4 inflammatory central nervous system conditions) 
Table 1 Demographic and clinical characteristics on admission

\begin{tabular}{|c|c|c|c|}
\hline Variable & $\begin{array}{l}\text { Patients with complete data } \\
(\mathrm{n}=224)\end{array}$ & Patients with missing data $(n=14)$ & $\begin{array}{l}\text { Difference between median } \\
\text { values }\end{array}$ \\
\hline Age, median (IQR), years & $52(38-60)$ & $54.5(43-61)$ & $p=0.29$ \\
\hline \multicolumn{4}{|l|}{ Gender, } \\
\hline Male, $n(\%)$ & $138(61.6 \%)$ & $7(50 \%)$ & \multirow[t]{2}{*}{$p=0.30$} \\
\hline Female, $\mathrm{n}(\%)$ & $86(38.4 \%)$ & $7(50 \%)$ & \\
\hline \multicolumn{4}{|l|}{ Affected limb* } \\
\hline Left, $n(\%)$ & $117(51.3 \%)$ & $8(57.1 \%)$ & \multirow[t]{2}{*}{$\mathrm{p}=0.39$} \\
\hline Right, $\mathrm{n}(\%)$ & $107(48.7 \%)$ & $6(42.9 \%)$ & \\
\hline Non dominant & $110(49.1 \%)$ & $7(50.0 \%)$ & \multirow[t]{2}{*}{$\mathrm{p}=0.87$} \\
\hline Dominant & $114(50.9 \%)$ & $7(50.0 \%)$ & \\
\hline $\begin{array}{l}\text { Median time since stroke } \\
\text { Months (IQR) }\end{array}$ & $18(12-51)$ & $16(12-22)$ & $p=0.29$ \\
\hline \multicolumn{4}{|l|}{ Modified Rankin scale, n (\%) } \\
\hline Median (IQR) & $2(2-3)$ & $2(2-2.75)$ & \multirow[t]{5}{*}{$p=0.32$} \\
\hline No significant disability & $20(9.9 \%)$ & $2(14.3 \%)$ & \\
\hline Slight disability & $106(47.4 \%)$ & $8(57.1 \%)$ & \\
\hline Moderate disability & $95(42.4 \%)$ & $4(28.6 \%)$ & \\
\hline Moderately severe & $3(1.3)$ & $0(0 \%)$ & \\
\hline The Barthel Index, median (IQR), Max=20 & $19(17-19)$ & $19(18-20)$ & $\mathrm{p}=0.12$ \\
\hline The Neurological Fatigue Index, median (IQR), Max=62 & $35(25-42)$ & $33(23-38)$ & $\mathrm{p}=0.34$ \\
\hline Hospital Anxiety and Depression Scale, median (IQR), Max=34 & $12(8-16)$ & $13(8.5-18)$ & $\mathrm{p}=0.64$ \\
\hline \multicolumn{4}{|l|}{ Sensory loss, $n, \%$} \\
\hline $0=$ Severe & $13(5.8 \%)$ & $2(14.3 \%)$ & \multirow[t]{3}{*}{$\mathrm{p}=0.36$} \\
\hline 1=Mild & $111(49.6 \%)$ & $7(50 \%)$ & \\
\hline $2=$ Normal & $100(44.6 \%)$ & $5(35.7 \%)$ & \\
\hline $\begin{array}{l}\text { Modified Fugl-Meyer } \\
\text { (upper limb), median (IQR) Max=54 }\end{array}$ & $26(16-37)$ & $27.5(19-34)$ & $\mathrm{p}=0.92$ \\
\hline Action Research Arm Test, median (IQR), Max=57 & $18(7-33)$ & $15(9.5-23)$ & $\mathrm{p}=0.92$ \\
\hline $\begin{array}{l}\text { Chedoke Arm and Hand Activity Inventory, median (IQR), } \\
\text { Max=91 }\end{array}$ & $40(28-55)$ & $33.5(27-40)$ & $\mathrm{p}=0.37$ \\
\hline $\begin{array}{l}\text { ArmA-A, } \\
\text { median (IQR), Max=28 }\end{array}$ & $8(5-12) \dagger$ & $8(6-12) \ddagger$ & $\mathrm{p}=0.55$ \\
\hline $\begin{array}{l}\text { ArmA-B, } \\
\text { median (IQR), Max=52 }\end{array}$ & $38(24-46) \dagger$ & $36(29-41) \ddagger$ & $p=0.65$ \\
\hline
\end{tabular}

Difference in medians tested with Wilcoxon signed-rank test. Difference in proportions was tested with $\chi^{2}$ test.

*202 (90.2\%) patients were right-handed, $22(9.8 \%)$ patients were left-handed.

†195 patients out of 224 had ArmA-A and ArmA-B measured on admission.

¥11 patients out of 14 had ArmA-A and ArmA-B measured on admission. *202 (90.2\%) patients were right-handed, 22 (9.8\%) patients were left-handed.

ArmA-A, Arm Activity Measure A; ArmA-B, Arm Activity Measure B.

and are excluded from this analysis. A total of 238 stroke patients were admitted, of whom 224 completed follow-up assessments at 6 weeks and 6 months after discharge from the programme. Of the 14 incomplete follow-ups, five patients considered it too far to travel for follow-up, four patients suffered intercurrent illness (recurrent stroke, fractured hip, complications of surgery, seizures) and five could not be contacted. Differences in median values between complete and missing data groups were tested using Wilcoxon signed-rank test. Where appropriate, differences in proportions between complete and missing data groups were tested using $\chi^{2}$ test. There were no differences in baseline characteristics between patients who completed follow-up and those who were lost to follow-up (table 1). Because (i) there was no systematic difference between subjects with complete data and those with missing data and (ii) only outcome data (not explanatory variables, which were collected at T1) were missing, then missing data were dealt with by performing a complete case analysis (ie, only using subjects where complete data were available).
Changes in upper limb deficit

The median scores for the affected upper limb at admission (T1), discharge (T2), 6 weeks (T3) and 6 months postdischarge (T4) are shown in table 2. The Friedman test was used to demonstrate a significant effect of time since admission for all outcome scores; FM-UL $\left(\chi^{2}(3)=431.8, \mathrm{p}<0.0001\right)$; ARAT

\begin{tabular}{lcccc}
\hline Table 2 & \multicolumn{4}{l}{ Upper limb scores before and after admission } \\
\hline & Admission & Discharge & 6 weeks & 6 months \\
\hline FM-UL & $26(16-37)$ & $34(23-44)$ & $35(23-46)$ & $37(24-48)$ \\
ARAT & $18(7-33)$ & $25(10-42)$ & $26(11-44)$ & $27(11-45)$ \\
CAHAI & $40(28-55)$ & $49(36-70)$ & $50(35-73)$ & $52(35-77)$ \\
ArmA-A & $8(4.5-12)$ & $5(2-8)$ & $4(2-7)$ & $3(1-6.5)$ \\
ArmA-B & $38(24-46)$ & $26(13-37)$ & $21(11-35)$ & $19(8.5-32)$ \\
\hline
\end{tabular}

All scores given as median (IQR).

ARAT, Action Research Arm Test; ArmA-A, Arm Activity Measure A; ArmA-B, Arm Activity Measure B; CAHAl, Chedoke Arm and Hand Activity Inventory; FM-UL, FuglMeyer (upper limb). 

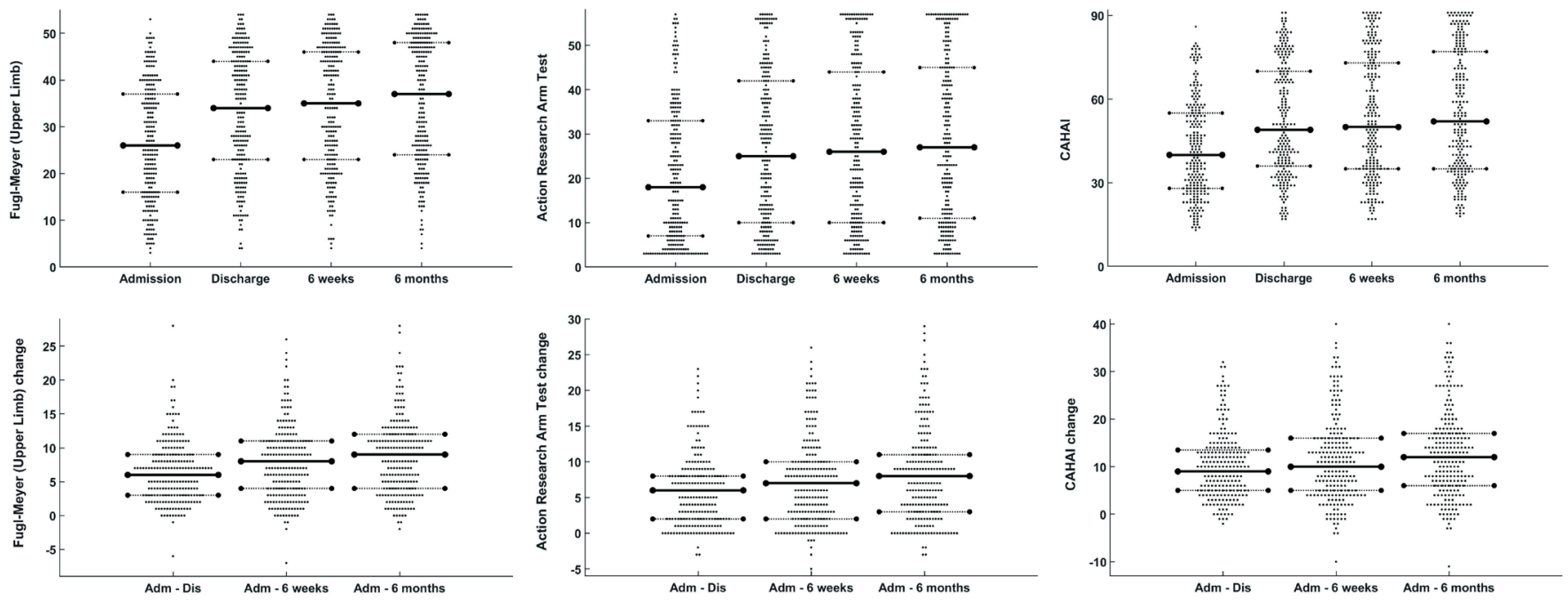

Figure 1 Outcome scores for all patients. Each data point represents a single patient. Top row shows individual scores at admission, discharge, 6 weeks and 6 months after discharge. Bottom row shows the individual difference scores for admission to discharge, admission to 6 weeks postdischarge and admission to 6 months postdischarge. Scores are shown for modified FM-UL, ARAT and CAHAl. Median (solid line) and upper and lower quartiles (dotted lines) are shown. ARAT, Action Research Arm Test; CAHAI, Chedoke Arm and Hand Activity Inventory; FM-UL, Fugl-Meyer (upper limb).

$\left(\chi^{2}(3)=383.2, \mathrm{p}<0.0001\right) ; \operatorname{CAHAI}\left(\chi^{2}(3)=371.6, \mathrm{p}<0.0001\right)$; $\operatorname{ArmA}-\mathrm{A}\left(\chi^{2}(3)=238.4, \mathrm{p}<0.0001\right)$ and $\operatorname{ArmA}-\mathrm{B}\left(\chi^{2}(3)=305.6\right.$, $\mathrm{p}<0.0001)$. Post-hoc analysis with Wilcoxon signed-rank test was conducted to test the hypothesis that the difference between the paired values $(\mathrm{T} 1-\mathrm{T} 2, \mathrm{~T} 1-\mathrm{T} 3, \mathrm{~T} 1-\mathrm{T} 4, \mathrm{~T} 2-\mathrm{T} 3, \mathrm{~T} 2-\mathrm{T} 4$, $\mathrm{T} 3-\mathrm{T} 4)$ comes from a distribution whose median is zero (ie, testing whether the change in score is significantly greater than zero). A Bonferroni correction was applied to the significance level for the six comparisons made for each of the five different outcome scores $(p<0.05 / 30=0.0017)$. For each outcome score, there was a significant difference between each pair of time points compared, except T2-T3 for the ArmA-A (online supplementary table). Individual scores at each time point are shown in figures 1 and 2. Individual changes in scores in comparison to admission are shown in table 3 and figures 1 and 2 . These results demonstrate improvement during the 3-week programme but also continued improvement after discharge.

By 6 months after the programme, $68.3 \%$ of patients had achieved greater than the MCID of 5.25 points on the FM-UL. For ARAT, this figure was $61.6 \%$. No published MCID is published for the other scores. MCID is often quoted as 10\% of the maximum score. If this were the case, then by 6 months, MCID would have been reached by $59.4 \%$ of patients for CAHAI, 53.8\% for ArmA-A and 72.3\% for ArmA-B.

\section{Predictors of outcome}

First, we were interested to see whether any baseline characteristics (T1) correlated with final outcome at T4. Spearman's rank correlation was used to show that admission values at $\mathrm{T} 1$ for FM-UL, ARAT, CAHAI, ArmA-A and ArmA-B all correlated with T4 values for FM-UL, ARAT, CAHAI, ArmA-A and ArmA-B. In addition, admission BI correlated with T4 CAHAI; admission mRS correlated with T4 FM-UL, ARAT and CAHAI; admission sensation correlated with T4 CAHAI. Age, NFI and HADS at $\mathrm{T} 1 \mathrm{did}$ not correlate with any scores at T4. All $\mathrm{p}$ values were corrected for multiple comparisons by Bonferroni correction (table 4). Figure 3 helps to visualise the influence of baseline admission scores on final score at 6-month follow-up. Furthermore, a Wilcoxon ranked sum test showed that T4 outcomes did not differ by gender or affected hemisphere. There was a trend for patients with nondominant affected limbs to achieve better (lower) ArmA-B scores in comparison to those with dominant affected limbs (median 17 for nondominant vs 27 for dominant, $\mathrm{p}=0.027$, uncorrected). There were no differences in $\mathrm{T} 4$ scores between patients with dominant or nondominant affected limbs for any of the other outcome scores.

Second, we were interested to see whether any baseline characteristics (T1) correlated with magnitude of change from admission to 6-months follow-up (T1-T4). Spearman's rank correlation was used to show that FM-UL, ARAT, CAHAI and ArmA-B at T1 correlated with change in ARAT and CAHAI (but not FM-UL) from T1 to T4. FM-UL at T1 also correlated with change in ArmA-B from T1 to T4. ArmA-A at T1 correlated with change in both CAHAI and ArmA-A from T1 to T4. ArmA-B at T1 correlated with change in both ArmA-A and ArmA-B from T1 to T4. Age, NFI, HADS, BI and mRS at T1 did not correlate with any scores at T4. All $\mathrm{p}$ values were corrected for multiple comparisons by Bonferroni correction (table 5). We also performed a Wilcoxon ranked sum test to show that change in scores from T1 to T4 was not different depending on gender or affected hemisphere. There was a trend for patients with nondominant affected limbs to improve on the ArmA-B between $\mathrm{T} 1$ and T4 to a greater degree than those with dominant affected limbs (median changes of -17.5 for nondominant vs -12 for dominant, $p=0.017$, uncorrected). There were no differences in change scores between patients with dominant or nondominant affected limbs for any of the other outcome scores.

We then performed multiple linear regression to look for predictors of either absolute scores at 6 months after treatment (T4) or the change in scores from admission to 6 months after treatment (T1-T4) using age, BI, mRS, NFI, HADS and sensation on admission together with the matching initial score on admission (eg, FM1 if attempting to predict FM4 or change from FM1 to FM4). Models predicting T4 scores for FM-UL, ARAT, CAHAI, ArmA-A and ArmA-B were all highly significant $(p<0.0001)$. Models predicting change from T1 to T4 for ARAT, CAHAI, ArmA-A and ArmA-B (but not FM-UL) were also highly significant $(\mathrm{p}<0.0001)$. However, the only factor that 

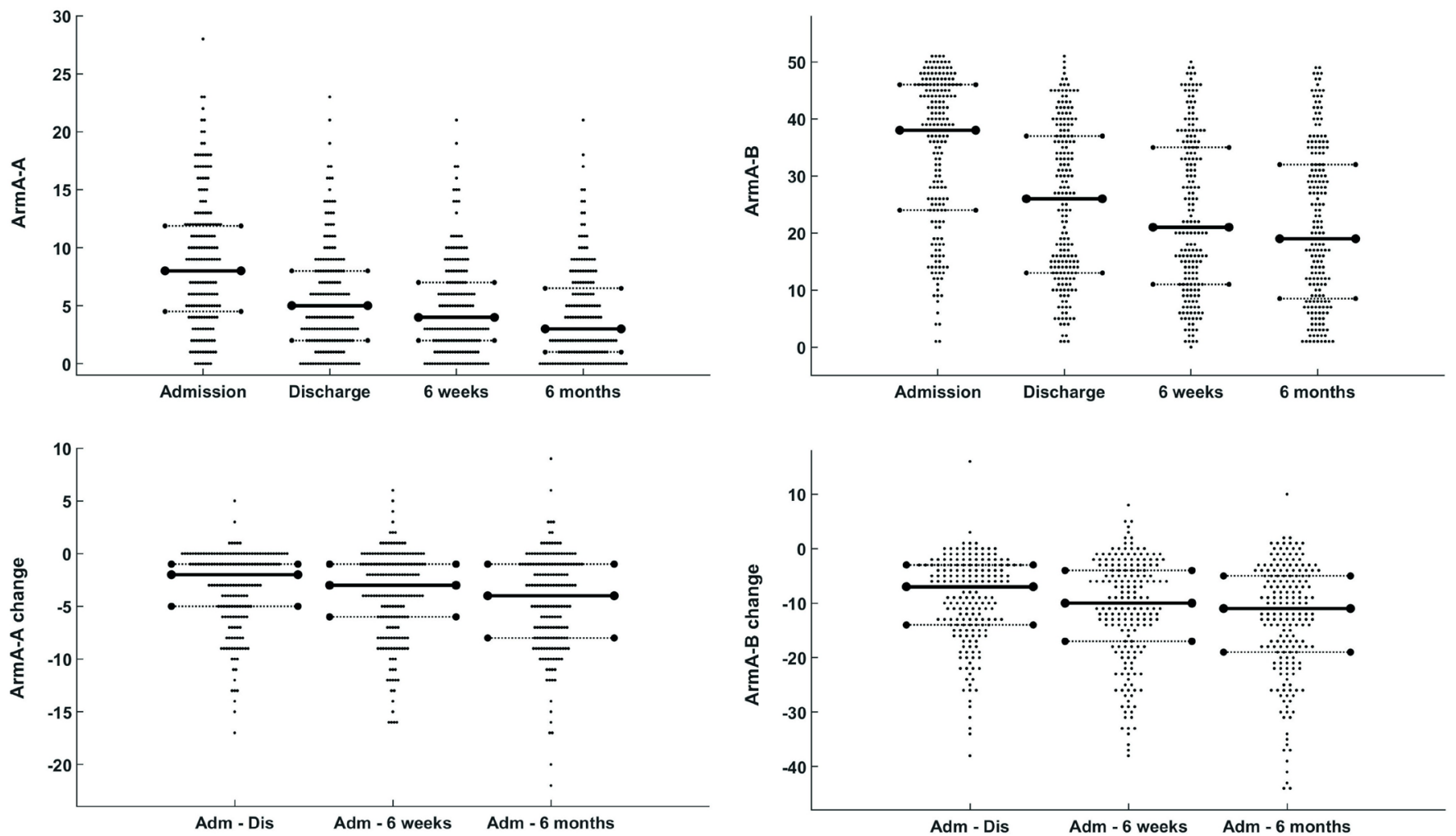

Figure 2 Self-reported outcome scores for all patients. Lower scores are better. Each data point represents a single patient. Top row shows individual scores at admission, discharge, 6 weeks and 6 months after discharge. Bottom row shows the individual difference scores for admission to discharge, admission to 6 weeks postdischarge and admission to 6 months postdischarge. Scores are shown for the ArmA-A and ArmA-B. Median (solid line) and upper and lower quartiles (dotted lines) are shown. ArmA-A, Arm Activity Measure A; ArmA-B, Arm Activity Measure B.

significantly contributed to these models was the matched initial score on admission (eg, ARAT at T1 predicting ARAT at T4), but not age, BI, mRS, NFI, HADS or sensation on admission. As such, this approach did not contribute further to the correlation results shown in tables 4 and 5, with regression models not able to account for a higher proportion of variance in scores at T4 or change in scores from T1 to T4 than the admission score (see also figure 3 ).

\section{Relationship between outcome measures}

Each outcome score assesses a different aspect of recovery. Using Spearman's rank correlation, we examined the relationship between the changes in outcome scores from admission to 6-month follow-up. A Bonferroni correction was applied to the significance level for the 10 comparisons made between the five different outcome scores $(\mathrm{p}<0.05 / 10=0.005)$ (table 6 and figure 4). Changes in FM-UL, ARAT and CAHAI correlated

Table 3 Changes in upper limb scores

\begin{tabular}{llcc}
\hline & Adm - Dis & Adm - 6 weeks & Adm - 6 months \\
\hline FM-UL & $6(3$ to 9$)$ & $8(4$ to 11$)$ & $9(4$ to 12$)$ \\
ARAT & $6(2$ to 8$)$ & $7(2$ to 10$)$ & $8(3$ to 11$)$ \\
CAHAI & $9(5$ to 13.5$)$ & $10(5$ to 16$)$ & $12(6$ to 17$)$ \\
ArmA-A & $-2(-5$ to -1$)$ & $-3(-6$ to -1$)$ & $-4(-8$ to -1$)$ \\
ArmA-B & $-7(-14$ to -3$)$ & $-10(-17$ to -4$)$ & $-11(-19$ to -5$)$ \\
\hline
\end{tabular}

All scores given as median (IQR).

ARAT, Action Research Arm Test; ArmA-A, Arm Activity Measure A; ArmA-B, Arm Activity Measure B; CAHAl, Chedoke Arm and Hand Activity Inventory; FM-UL, FuglMeyer (upper limb). weakly with one another. The ArmA-B change correlated with changes in both ArmA-A and ARAT.

\section{DISCUSSION}

We report the results from a single-centre clinical service delivering high dose and intensity of upper limb focused neurorehabilitation for people with stroke. Almost all of these patients were in the chronic ( $>6$ months after stroke) phase. The key messages

Table 4 Correlation between baseline scores and 6-month outcomes

\begin{tabular}{llllll}
\hline & $\begin{array}{l}\text { FM-UL } \\
\text { (T4) }\end{array}$ & $\begin{array}{l}\text { ARAT } \\
\text { (T4) }\end{array}$ & $\begin{array}{l}\text { CAHAI } \\
\text { (T4) }\end{array}$ & $\begin{array}{l}\text { ArmA-A } \\
\text { (T4) }\end{array}$ & $\begin{array}{l}\text { ArmA-B } \\
\text { (T4) }\end{array}$ \\
\hline Age & n.s. & n.s. & n.s. & n.s. & n.s. \\
\hline Barthel Index & n.s. & n.s. & 0.28 & n.s. & n.s. \\
modified Rankin Scale & -0.29 & -0.25 & -0.32 & n.s. & n.s. \\
\hline Neurological Fatigue Index & n.s. & n.s. & n.s. & n.s. & n.s. \\
Hospital Anxiety and & n.s. & n.s. & n.s. & n.s. & n.s. \\
Depression Scale & & & & & \\
Sensation & n.s. & n.s. & 0.22 & n.s. & n.s. \\
FM-UL (T1) & 0.92 & 0.84 & 0.83 & -0.42 & -0.63 \\
\hline ARAT (T1) & 0.87 & 0.95 & 0.91 & -0.48 & -0.71 \\
\hline CAHAI (T1) & 0.84 & 0.88 & 0.92 & -0.46 & -0.71 \\
\hline ArmA-A (T1) & -0.39 & -0.44 & -0.44 & 0.56 & 0.41 \\
ArmA-B (T1) & -0.53 & -0.58 & -0.61 & 0.40 & 0.71 \\
\hline
\end{tabular}

Correlation coefficient (rho) given only when significant at $\mathrm{p}<0.05$ corrected for 55 multiple comparisons $(p<0.0009)$. n.s. $=$ not significant.

ARAT, Action Research Arm Test; ArmA-A, Arm Activity Measure A; ArmA-B, Arm Activity Measure B; CAHAl, Chedoke Arm and Hand Activity Inventory; FM-UL, FuglMeyer (upper limb). 

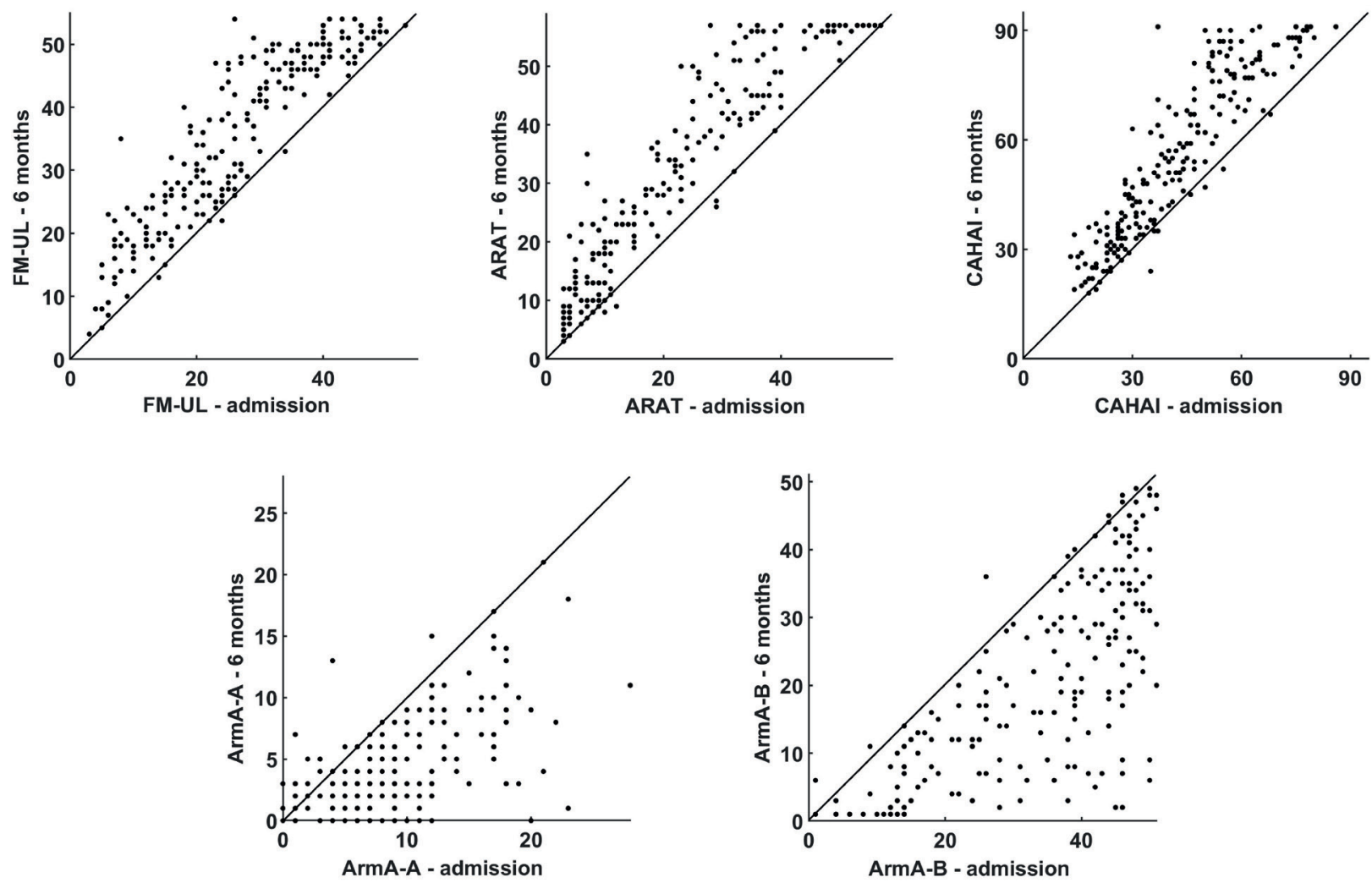

Figure 3 Admission score plotted against final scores at 6 months postdischarge for FM-UL, ARAT, CAHAl, ArmA-A and ArmA-B. The black line represents the line of 'no change'. Patients above this line for FM-UL, ARAT and CAHAI have improved. Patients below this line for ArmA-A and ArmA-B have improved. FM-UL, Fugl-Meyer (upper limb); ARAT, Action Research Arm Test; CAHAl, Chedoke Arm and Hand Activity Inventory; ArmA-A, Arm Activity Measure A; ArmA-B, Arm Activity Measure B.

from our results are as follows: (i) patients were able to complete the 90 hours of the programme, despite exhibiting a range of impairments and fatigue levels, (ii) large clinical improvements in upper limb impairment (FM-UL) and activity (ARAT, CAHAI and ArmA) were observed and (iii) these changes were maintained, or even improved on, 6 months after treatment.

The QSUL programme is based on the hypothesis that a high dose and intensity of upper limb neurorehabilitation can lead to large clinically meaningful improvements in chronic stroke patients. It is worth comparing the magnitude of the changes with previous reported clinical trials of upper limb rehabilitation. Most obviously, the study by McCabe et al ${ }^{10}$ investigated the effects of 300 hours of upper limb rehabilitation in 48 chronic stroke patients with similar characteristics to those reported here (baseline FM-UL 24, compared with 26 in our patients) and reported an increase of $8-11$ points on the FM-UL

Table 5 Correlation between baseline scores and change scores from admission to 6 months

\begin{tabular}{|c|c|c|c|c|c|}
\hline & FM-UL (T1-T4) & ARAT (T1-T4) & CAHAI (T1-T4) & ArmA-A (T1-T4) & ArmA-B (T1-T4) \\
\hline Age & n.s. & n.s. & n.s. & n.s. & n.s. \\
\hline Barthel Index & n.s. & n.s. & n.s. & n.s. & n.s. \\
\hline Neurological Fatigue Index & n.s. & n.s. & n.s. & n.s. & n.s. \\
\hline Hospital Anxiety and Depression Scale & n.s. & n.s. & n.s. & n.s. & n.s. \\
\hline Sensation & n.s. & n.s. & n.s. & n.s. & n.s. \\
\hline CAHAI (T1) & n.s. & 0.42 & 0.35 & n.s. & n.s. \\
\hline ArmA-A (T1) & n.s. & n.s. & -0.31 & -0.72 & n.s. \\
\hline ArmA-B (T1) & n.s. & -0.26 & -0.38 & -0.28 & -0.26 \\
\hline
\end{tabular}

Correlation coefficient (rho) given only when significant at $p<0.05$ corrected for 55 multiple comparisons ( $<<0.0009)$. n.s. $=$ not significant.

ARAT, Action Research Arm Test; ArmA-A, Arm Activity Measure A; ArmA-B, Arm Activity Measure B; CAHAl, Chedoke Arm and Hand Activity Inventory; FM-UL, Fugl-Meyer (upper $\operatorname{limb}$. 


\begin{tabular}{llllll}
\hline Table 6 & \multicolumn{5}{c}{ Correlations between changes in different outcome scores } \\
\hline & $\begin{array}{l}\text { FM-UL } \\
\text { (T1-T4) }\end{array}$ & ARAT (T1-T4) & $\begin{array}{l}\text { CAHAl } \\
\text { (T1-T4) }\end{array}$ & $\begin{array}{l}\text { ArmA-A } \\
\text { (T1-T4) }\end{array}$ & $\begin{array}{l}\text { ArmA-B } \\
\text { (T1-T4) }\end{array}$ \\
\hline FM-UL (T1-T4) & - & 0.32 & 0.34 & n.s. & n.s. \\
\hline ARAT (T1-T4) & 0.32 & - & 0.37 & n.s. & -0.25 \\
CAHAI (T1-T4) & 0.34 & 0.37 & - & n.s. & -0.20 \\
ArmA-A(T1-T4) & n.s. & n.s. & n.s. & - & 0.29 \\
ArmA-B (T1-T4) & n.s. & -0.25 & -0.20 & 0.29 & - \\
\hline
\end{tabular}

Correlation coefficient (rho) given only when significant at $p<0.05$ corrected for 10 multiple comparisons $(p<0.005)$. n.s. $=$ not significant.

ARAT, Action Research Arm Test; ArmA-A, Arm Activity Measure A; ArmA-B, Arm Activity Measure B; CAHAl, Chedoke Arm and Hand Activity Inventory; FM-UL, FuglMeyer (upper limb).

at the end of 300 hours of upper limb treatment (although later follow-up results have not been reported). In the QSUL programme, the median FM-UL (modified) improvement was 6 points after the 90 hours programme, increasing to 9 points by 6 -months follow-up, achieving a similar overall magnitude of change in comparison to McCabe et al. In addition to reductions in impairment (FM-UL), our patients also improved at the level of activity (ARAT and CAHAI) and participation (using the patient-reported outcome measure, ArmA). Importantly, we report that these group-level effects continued to improve at 6 weeks and then 6 months after treatment had stopped, (figures 1 and 2).

Other than the study by McCabe et al, the general view has been that large changes, especially in impairment, have not been seen for behavioural interventions of the post-stroke upper limb. ${ }^{3}$ Direct comparison with other studies is difficult because some may have been conducted in less severely affected patients, the dose (measured in time) of the intervention may have been lower and the numbers of patients enrolled has been much lower than we report here. While this report of our single-centre clinical outcomes is not a replication of the McCabe study, it suggests that the next wave of upper limb rehabilitation trials must investigate much higher doses of the treatment than is currently attempted. While we will hear the refrain that it is not possible to deliver such high doses in current healthcare settings (the pragmatic view), it is in fact precisely the role of clinical research to challenge what we currently do in order to reshape and improve our clinical services to make them better (the aspirational view). Those involved in stroke medicine will recognise that this is exactly the effect that thrombolysis had on acute management of stroke, leading to the high-quality hyperacute stroke services we see today. Post-stroke neurorehabilitation must adopt the same aspirational approach. ${ }^{20}$

While the appropriate dose of upper limb rehabilitation is debated, the content of the intervention receives less attention. Our approach was based on analysis of movement and performance in activities of daily living, reduction of impairment and re-education of quality and control of movement within activities of daily living, all performed in an iterative and nonlinear fashion. Education, self-efficacy and goal setting were integral components of the programme, and we can speculate that the education and focus on self-efficacy contributed to the continued increase in scores after treatment stopped. The therapeutic approach taken towards specific impairments was the same across patients, but approaches at the activity and participation level will necessarily vary as they are tailored to an individual's specific meaningful goals. We acknowledge that an immediate challenge for us and for the field in general is to be able to define what the 'active ingredients' of neurorehabilitation are, so that these elements can be further tested. Our approach appears to have similarities with that taken by McCabe et al, with an emphasis on the quality of the movement as well as the final goal of the movement. The latter is often the sole focus of task-specific repetition, which has been used in many upper limb rehabilitation studies to date. The evidence for repetitive task-specific training is of low to moderate quality ${ }^{21}$ and the effects have been disappointingly small even when the number of repetitions has been greatly increased. ${ }^{8}$ It is difficult to tell whether this lack of effect is specific to task-specific training because in general the dose (in terms of hours of active training) has been quite low, whether delivered by a therapist ${ }^{21}$ or robotic device. ${ }^{22}$ There have been suggestions that chronic stroke patients might not tolerate more training than has been offered in these trials, ${ }^{8}$ but the study of McCabe et $a l^{10}$ and now our own clinical experience undermine this view.

Although this result is exciting, we must acknowledge that it is a single-centre clinical service with no randomisation and no control group. Although there are some reports of late improvement up to 12 months after lacunar stroke, ${ }^{23}$ we expect that chronic stroke patients, who are often several years after their stroke, will not change across this range of outcome scores without treatment. We suggest that it is unlikely that this magnitude of change would be seen without therapeutic intervention, but this
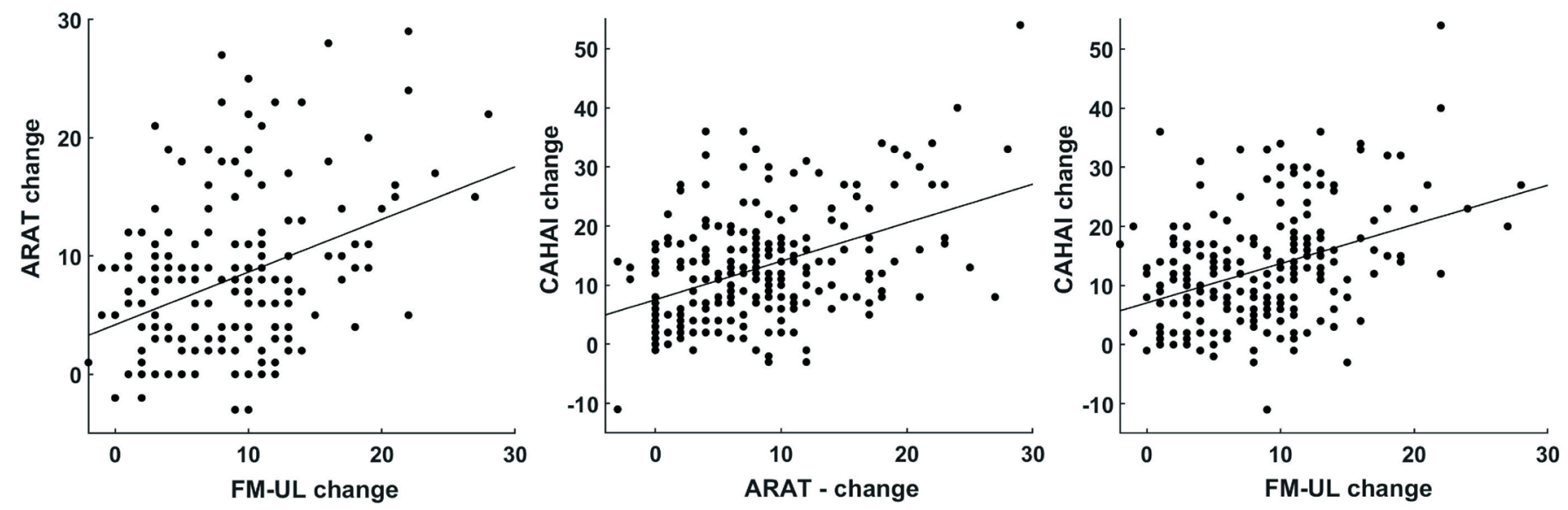

Figure 4 Change in scores from admission to 6 months postdischarge for FM-UL, ARAT, CAHAl, plotted against each other. ARAT, Action Research Arm Test; CAHAl, Chedoke Arm and Hand Activity Inventory; FM-UL, Fugl-Meyer (upper limb). 
assertion requires empirical confirmation. Our work, however, provides strong justification for undertaking such high-dose clinical trials. Clinic data on which to base these future studies are sorely lacking and this has led to the proliferation of low-dose pragmatic rehabilitation studies. However, there is still much to be learnt from published data arising from clinical services in neurorehabilitation, ${ }^{24}$ making up for lack of clinical trial rigour with large sample sizes that are rarely achieved in clinical trials. Data from clinical services therefore have the potential to refine what experimental questions are addressed in future randomised clinical trials. Another limitation is the unblinded nature of the assessments, introducing possible bias into the results. All assessors are also therapists on the programme, although we ensure that assessments are carried out by therapists from another team who are not treating the patient. Fidelity of outcome scores is always an important issue in clinical trials. In our programme, we have instituted regular in-service training for FM-UL, ARAT and CAHAI to ensure high levels of reproducibility between assessors.

A common complaint regarding single-centre clinical services is that they select only patients most likely to succeed. It is important to acknowledge that the QSUL programme does not have strict inclusion/exclusion criteria. Although our patients are generally younger than the average stroke patient and have high levels of independence, they have a wide range of upper limb impairments. It can be seen from figures 1 and 2 that the range of severity of the upper limb on the QSUL programme was very broad, quite unlike a clinical trial, although the median scores suggest that patients were weighted towards the moderatesevere end of upper limb impairment and activity limitation. We would argue that the QSUL programme is aspirational in its goals (high-quality, high-dose, high-intensity treatment) and pragmatic in its application (wide range of patients admitted onto the programme). It is interesting to consider the potential impact of initial upper limb severity on our outcomes, but in fact, although initial score correlates well with the final score at 6 months (table 4), the change in score correlates only weakly with initial score (table 5, figure 3 ). There are two ways this 'mixed-bag' approach may have potentially reduced the overall magnitude of the clinical effects we observed. First, there may be a ceiling effect from patients with relatively high scores on admission. Second, many of our patients were quite severely impaired. Indeed, a quarter of our patients had an admission ARAT below 7, which suggests they have great difficulty in using their hand in any kind of activity of daily living. The treatment goals in these type of patients might initially be very different to the moderate-mild type patients, with benefits less likely to be reflected in changes in FM-UL and ARAT compared with the CAHAI or ArMA, for example. However, the wide range of patients treated in the programme means that no clear patterns, in relation to differential effects on various scores, could be seen (table 6, figure 4).

Some might argue that the wide range of responses, including those patients who changed very little, points to the need for stratification and selection of those patients most likely to respond, and this is certainly a reasonable view. However, the first point to make is that our results show that with large enough numbers and a high enough dose of the intervention, it is possible to demonstrate large group-level effects in relatively unselected populations of chronic stroke patients. Indeed, if the former (stratification) approach is to be adopted, then these kinds of large data sets will be required to determine what the characteristics of the 'responders' are likely to be. The second point to make is that we found no strong clinical indicators, among our measured explanatory variables, of which patients were most likely to change, including sensory loss in the hand, fatigue ${ }^{25}$ and depression (tables 4 and 5). It might be that neurophysiological (presence or absence of motor evoked potentials), ${ }^{26}$ neuroimaging (assessment of corticospinal tract, whole brain damage or brain function) ) $^{27-30}$ or cognitive (sustained attention, memory, motivation $)^{3132}$ measures are required for accurate stratification based on likely response, and this can certainly be tested in future.

In summary, here we present the results from a single-centre clinical service dedicated to post-stroke upper limb neurorehabilitation in chronic stroke patients. Despite treating patients with a wide range of impairment, we were able to show a large group-level magnitude of change at both the impairment and activity level. Our experience suggests that much higher doses and intensity of upper limb neurorehabilitation can be delivered and our results should inform future clinical trial design.

Acknowledgements Thanks to all the physiotherapists and occupational therapists at The National Hospital for Neurology and Neurosurgery, Queen Square, who have treated patients on this programme. Thanks to UCLH Charities, Friends of UCLH and The National Brain Appeal for funding to purchase equipment used in this programme.

Contributors All authors made substantial contributions to the conception of the work and interpretation of the data. Analysis was performed by NW. All authors were involved in drafting and revising the work critically for important intellectual content. All authors provided final approval of the version published. All authors agree to be accountable for all aspects of the work in ensuring that questions related to the accuracy or integrity of any part of the work are appropriately investigated and resolved.

Funding The authors have not declared a specific grant for this research from any funding agency in the public, commercial or not-for-profit sectors.

Competing interests None declared.

Patient consent for publication Not required.

Provenance and peer review Not commissioned; externally peer reviewed.

\section{REFERENCES}

1 Licher S, Darweesh SKL, Wolters FJ, et al. Lifetime risk of common neurological diseases in the elderly population. J Neurol Neurosurg Psychiatry 2019;90.

2 Broeks JG, Lankhorst GJ, Rumping K, et al. The long-term outcome of arm function after stroke: results of a follow-up study. Disabil Rehabil 1999;21:357-64.

3 Krakauer JW, Carmichael ST, Corbett D, et al. Getting neurorehabilitation right: what can be learned from animal models? Neurorehabil Neural Repair 2012;26:923-31.

4 Lohse KR, Lang CE, Boyd LA. Is more better? Using metadata to explore doseresponse relationships in stroke rehabilitation. Stroke 2014;45:2053-8.

5 Langhorne P, Coupar F, Pollock A. Motor recovery after stroke: a systematic review. Lancet Neurol 2009:8:741-54.

6 Winstein CJ, Wolf SL, Dromerick AW, et al. Effect of a Task-Oriented rehabilitation program on upper extremity recovery following motor stroke: the ICARE randomized clinical trial. JAMA 2016;315:571-81.

7 Lo AC, Guarino PD, Richards LG, et al. Robot-assisted therapy for long-term upperlimb impairment after stroke. N Engl J Med 2010;362:1772-83.

8 Lang CE, Strube MJ, Bland MD, et al. Dose response of task-specific upper limb training in people at least 6 months poststroke: a phase II, single-blind, randomized, controlled trial. Ann Neurol 2016:80:342-54.

9 Klamroth-Marganska V, Blanco J, Campen K, et al. Three-dimensional, task-specific robot therapy of the arm after stroke: a multicentre, parallel-group randomised trial. Lancet Neurol 2014;13:159-66.

10 McCabe J, Monkiewicz M, Holcomb J, et al. Comparison of robotics, functional electrical stimulation, and motor learning methods for treatment of persistent upper extremity dysfunction after stroke: a randomized controlled trial. Arch Phys Med Rehabil 2015:96:981-90.

11 Duncan PW, Propst M, Nelson SG. Reliability of the Fugl-Meyer assessment of sensorimotor recovery following cerebrovascular accident. Phys Ther 1983;63:1606-10.

12 Fugl-Meyer AR, Jääskö L, Leyman I, et al. The post-stroke hemiplegic patient. 1. a method for evaluation of physical performance. Scand J Rehabil Med 1975;7:13-31.

13 Crow JL, Harmeling-van der Wel BC. Hierarchical properties of the motor function sections of the Fugl-Meyer Assessment Scale for people after stroke: a retrospective study. Phys Ther 2008;88:1554-67. 
14 Hsieh Y-W, Hsueh I-P, Chou Y-T, et al. Development and validation of a short form of the Fugl-Meyer motor scale in patients with stroke. Stroke 2007;38:3052-4.

15 Page SJ, Fulk GD, Boyne P. Clinically important differences for the upper-extremity Fugl-Meyer scale in people with minimal to moderate impairment due to chronic stroke. Phys Ther 2012;92:791-8.

16 Platz T, Pinkowski C, van Wijck F, et al. Reliability and validity of arm Function assessment with standardized guidelines for the Fugl-Meyer test, action research arm test and box and block test: a multicentre study. Clin Rehabil 2005;19:404-11.

17 Van der Lee JH, De Groot V, Beckerman H, et al. The intra- and interrater reliability of the action research arm test: a practical test of upper extremity function in patients with stroke. Arch Phys Med Rehabil 2001;82:14-19.

18 Barreca SR, Stratford PW, Lambert CL, et al. Test-retest reliability, validity, and sensitivity of the Chedoke arm and hand activity inventory: a new measure of upperlimb function for survivors of stroke. Arch Phys Med Rehabil 2005;86:1616-22.

19 Ashford S, Turner-Stokes L, Siegert R, et al. Initial psychometric evaluation of the arm activity measure (armA): a measure of activity in the hemiparetic arm. Clin Rehabil 2013;27:728-40.

20 Bernhardt J, Borschmann K, Boyd L, et al. Moving rehabilitation research forward: developing consensus statements for rehabilitation and recovery research. Int I Stroke 2016;11:454-8.

21 French $\mathrm{B}$, Thomas $\mathrm{LH}$, Coupe J, et al. Repetitive task training for improving functional ability after stroke. Cochrane Database Syst Rev 2016;19.

22 Veerbeek JM, Langbroek-Amersfoort AC, van Wegen EE, et al. Effects of robot-assisted therapy for the upper limb after stroke. Neurorehabil Neural Repair 2017;31:107-21.
23 Ganesh A, Gutnikov SA, Rothwell PM, et al. Late functional improvement after lacunar stroke: a population-based study. J Neurol Neurosurg Psychiatry 2018;89:1301-7.

24 Freeman JAet al. Evaluating neurorehabilitation: lessons from routine data collection. Journal of Neurology, Neurosurgery \& Psychiatry 2005;76:723-8.

25 De Doncker W, Dantzer R, Ormstad H, et al. Mechanisms of poststroke fatigue. J Neurol Neurosurg Psychiatry 2018;89:287-93.

26 Agarwal S, Koch G, Hillis AE, et al. Interrogating cortical function with transcranial magnetic stimulation: insights from neurodegenerative disease and stroke. J Neurol Neurosurg Psychiatry 2019;90.

27 Aguilar OM, Kerry SJ, Ong YH, et al. Lesion-site-dependent responses to therapy after aphasic stroke. J Neurol Neurosurg Psychiatry 2018;89:1352-4.

28 Park CH, Kou N, Ward NS. The contribution of lesion location to upper limb deficit after stroke. J Neurol Neurosurg Psychiatry 2016;87:1283-6.

29 Rondina JM, Park CH, Ward NS. Brain regions important for recovery after severe poststroke upper limb paresis. J Neurol Neurosurg Psychiatry 2017;88:737-43.

30 Burke Quinlan E, Dodakian L, See J, et al. Neural function, injury, and stroke subtype predict treatment gains after stroke. Ann Neurol 2015;77:132-45.

31 Robertson IH, Ridgeway V, Greenfield E, et al. Motor recovery after stroke depends on intact sustained attention: a 2-year follow-up study. Neuropsychology 1997;11:290-5.

32 Quattrocchi G, Greenwood R, Rothwell JC, et al. Reward and punishment enhance motor adaptation in stroke. J Neurol Neurosurg Psychiatry 2017;88:730-6. 